\title{
Spatial and spatio-temporal patterns in a cell-haptotaxis model
}

\author{
P. K. Maini \\ Department of Mathematics, University of Utah, Salt Lake City, UT 84112, USA
}

\begin{abstract}
We investigate a cell-haptotaxis model for the generation of spatial and spatio-temporal patterns in one dimension. We analyse the steady state problem for specific boundary conditions and show the existence of spatially hetero-geneous steady states. A linear analysis shows that stability is lost through a Hopf bifurcation. We carry out a nonlinear multi-time scale perturbation procedure to study the evolution of the resulting spatio-temporal patterns. We also analyse the model in a parameter domain wherein it exhibits a singular dispersion relation.
\end{abstract}

Key words: Morphogenesis - Spatio-temporal pattern - Mechanochemical - Haptotaxis - Singular dispersion relation

\section{Introduction}

Several models have been proposed to describe cell pattern formation in early embryonic development. A large number of these models are based on reaction diffusion systems involving chemicals which, in the appropriate parameter domain, bifurcate from a homogeneous steady state and evolve into a heterogeneous steady state (see, for example, [1-3]). This chemical pre-pattern is then interpreted by cells which differentiate accordingly to form a cell pattern [4].

Recently, an alternative approach to embryological pattern formation has been proposed by Murray, Oster and their co-workers $[5,6]$ based on the mechanochemical interaction of cells with their surrounding tissue. Linear analysis of such models suggests that they exhibit wide-ranging pattern formation capability [7]. Perelson et al. [8] confirmed this by numerically solving the nonlinear system in one dimension to get spatially heterogeneous and spatiotemporally oscillating patterns. Maini and Murray [9] carried out a nonlinear analysis for a simple version of the model to investigate spatial pattern formation. 
In this paper we analyse a more complicated version of the model to determine possible spatial patterns and spatio-temporally oscillating solutions. In Sect. 2 we briefly motivate the model equations. In Sect. 3 we investigate the linear stability of the non-trivial steady state and show that it is unstable to perturbations in a certain range of wave numbers. In Sect. 4 we investigate the possible spatial patterns the model can exhibit. In Sect. 5 we consider periodic boundary conditions. Under these conditions the spatially uniform steady state loses stability through a Hopf bifurcation and we examine the evolution of such spatio-temporally oscillating solutions using the method of multi-time scale perturbation (see, for example, [10]). In Sect. 6, we investigate a singular dispersion relation that arises in a certain parameter domain for the model. We analyse the subsequent fast-focussing problem and show the possible existence of small amplitude spatial pattern.

\section{The model}

In 1983, Oster et al. [5] proposed a mechanical model for pattern formation based on mesenchymal cells moving through and deforming an extracellular matrix (ECM). In this section we briefly describe a simplified version of the full model and refer the reader to the original paper for full details. The model is based on the three field variables:

$n(\underline{x}, t)=$ density of mesenchymal cells at position $\underline{x}$ and time $t$,

$\rho(\underline{x}, t)=$ density of ECM at position $\underline{x}$ and time $t$,

$\underline{u}(\underline{x}, t)=$ displacement at time $t$ of a material point of ECM initially at $\underline{x}$.

The model consists of three equations:

Cell conservation.

$$
\frac{\partial n}{\partial t}=-\nabla \cdot \underline{J}
$$

where $\underline{J}$ is a flux term. Several cell processes contribute to this term. We shall focus on three in particular, namely, diffusion, convection and haptotaxis.

The diffusive flux is of Fickian type,

$$
\underline{J}_{D}=-D \nabla n
$$

where $D$ is the positive diffusion coefficient.

The convective flux is due to cells riding passively on the ECM and may be modeled by

$$
\underline{J}_{C}=n \frac{\partial \underline{u}}{\partial t} .
$$

Cells move by attaching their filopodia to specialized adhesive sites in the ECM and move up a gradient in adhesive site density. This process is called haptotaxis. 
Assuming that adhesive site density is proportional to ECM density, the haptotactic flux may be modeled by

$$
\underline{J}_{H}=\alpha n \nabla \rho,
$$

where $\alpha$ is the positive haptotactic coefficient. Thus the cell conservation equation is

$$
\frac{\partial n}{\partial t}=D \nabla^{2} n-\alpha \nabla \cdot(n \nabla \rho)-\nabla \cdot\left(n \frac{\partial \underline{u}}{\partial t}\right),
$$

where we have assumed $D$ and $\alpha$ to be constants for simplicity.

Matrix conservation. Assuming that at the stage of pattern formation the cells have stopped secreting matrix the matrix equation is

$$
\frac{\partial \rho}{\partial t}+\nabla \cdot\left(\rho \frac{\partial \underline{u}}{\partial t}\right)=0
$$

where we assume that matrix moves only by convection.

Mechanical force balance. We are in the regime of low Reynolds number so viscoelastic forces dominate inertial terms and cell motion instantly ceases when the applied forces switch off. Therefore, the mechanical force balance equation is

$$
\nabla \cdot \underline{\sigma}+\rho \underline{F}=\underline{0},
$$

where $\underline{\sigma}$ is the stress tensor and $\underline{F}$ the body force. Modeling the cell-matrix composite as a linear, isotropic, viscoelastic material, the stress tensor may be written as

$$
\underline{\sigma}=\underline{\sigma}_{\text {matrix }}+\underline{\sigma}_{\text {cell-matrix }}
$$

where

$$
\begin{gathered}
\underline{\sigma}_{\text {matrix }}=\mu_{1} \frac{\partial \underline{e}}{\partial t}+u_{2} \frac{\partial \Theta}{\partial t} I+\frac{E}{1+v}\left[\underline{e}+v^{*} \Theta I\right] \\
\text { viscous }
\end{gathered}
$$

$\Theta=\nabla \cdot \underline{u}$, the dilation, $\underline{e}=\frac{1}{2}\left[\nabla \underline{u}+\nabla \underline{u}^{T}\right]$, the linear strain tensor (superscript $T$ denotes the transpose), $\mu_{1}$ and $\mu_{2}$ are the shear and bulk viscosities respectively, $v$ the Poisson ratio, $v^{*}=1 /(1-2 v)$, and $I$ is the unit tensor. The stress exerted by the contractile forces of the cells on the matrix is assumed to be of the form

$$
\underline{\sigma}_{\text {cell-matrix }}=\tau n \rho I
$$

where $\tau$ is the positive traction coefficient. Assuming that the cell-matrix composite is subject to a linear restoring force, $\underline{F}=-s \underline{u}$, where $s$ is a positive constant. Thus the mechanical balance equation is

$$
\nabla \cdot\left[\mu_{1} \frac{\partial \underline{e}}{\partial t}+\mu_{2} \frac{\partial \Theta}{\partial t} \boldsymbol{I}+\left\{\frac{E}{1+v}\right\}\left(\underline{e}+v^{*} \Theta \boldsymbol{I}\right)+\tau n \rho \boldsymbol{I}\right]=s \underline{u} \rho .
$$

Equations (2.1), (2.2) and (2.3) are simplified versions of the model presented in 
[5]. The system may be non-dimensionalized and, with appropriate non-dimensionalization (see Appendix A.1), the one-dimensional version of the model is

$$
\begin{gathered}
\frac{\partial n}{\partial t}+\frac{\partial}{\partial x}\left(n \frac{\partial u}{\partial t}\right)=D \frac{\partial^{2} n}{\partial x^{2}}-\alpha \frac{\partial}{\partial x}\left(n \frac{\partial \rho}{\partial x}\right) \\
\mu \frac{\partial^{3} u}{\partial x^{2} \partial t}+\frac{\partial^{2} u}{\partial x^{2}}+\tau \frac{\partial}{\partial x}(n \rho)=s u \rho \\
\frac{\partial \rho}{\partial t}+\frac{\partial}{\partial x}\left(\rho \frac{\partial u}{\partial t}\right)=0
\end{gathered}
$$

where $x$ is now the one-dimensional spatial coordinate and $u(x, t)$ the displacement of a material point initially at $x, \mu=\mu_{1}+\mu_{2}$, and $n=\rho=1, \mu=0$ is the biologically relevant steady state. In this paper we shall restrict our attention to the one-dimensional case.

We simplify the system further by linearising $(2.4 \mathrm{c})$ about the steady state and integrating, to give

$$
\rho \approx 1-\frac{\partial u}{\partial x}
$$

Substituting $(2.5)$ into $(2.4 \mathrm{a}, \mathrm{b})$ gives

$$
\begin{gathered}
\frac{\partial n}{\partial t}+\frac{\partial}{\partial x}\left(n \frac{\partial u}{\partial t}\right)=D \frac{\partial^{2} n}{\partial x^{2}}+\alpha \frac{\partial}{\partial x}\left(n \frac{\partial^{2} u}{\partial x^{2}}\right) \\
\mu \frac{\partial^{3} u}{\partial x^{2} \partial t}+\frac{\partial^{2} u}{\partial x^{2}}+\tau \frac{\partial}{\partial x}\left(n-n \frac{\partial u}{\partial x}\right)=s u\left(1-\frac{\partial u}{\partial x}\right)
\end{gathered}
$$

with the condition

$$
\frac{\partial u}{\partial x}<1
$$

Note that the condition (2.6c) is forced by (2.5) since $\rho$ cannot be negative. This assumption is consistent with the derivation of the model equations which was was based on a small strain approximation. The pair of equations $(2.6 a, b)$ is the version of the model that we will study. We shall discuss boundary conditions in Section 4.

\section{Linear stability analysis}

The pair of Eqs. (2.6a, b) has the non-trivial steady state $n=1, u=0$. We wish to analyse the linear stability of this uniform steady state to small spatial perturbations. For smooth initial data the solution to the linearised problem may be written as the integral of functions of the form $e^{\sigma t+i k x}$ over $k$, where $\sigma(k)$ is the temporal growth rate of the Fourier component of the initial perturbation which has wave number $k$. To analyse the temporal growth of these Fourier 
components (see, for example, [11]) we look for solutions of the form

$$
\left(\begin{array}{l}
n-1 \\
u
\end{array}\right) \alpha e^{\sigma t+i k x},
$$

to the linearised system and we obtain the dispersion relation

$$
\mu k^{2} \sigma^{2}\left(k^{2}\right)+b\left(k^{2}\right) \sigma\left(k^{2}\right)+c\left(k^{2}\right)=0,
$$

where

$$
\begin{aligned}
& b\left(k^{2}\right)=\mu D k^{4}+(1-2 \tau) k^{2}+s, \\
& c\left(k^{2}\right)=\{D-(D+\alpha) \tau\} k^{4}+s D k^{2} .
\end{aligned}
$$

The steady state $n=1, u=0$ will be unstable if there exists a wave number $k$ such that $R l\left(\sigma\left(k^{2}\right)\right)$ is greater than zero, that is, the linearized system will extract the $k$ wave number from the Fourier decomposition of the initial perturbation and cause it to grow. It is easy to show that if $D>(D+\alpha) \tau$ then the uniform steady state loses stability as $\tau$ increases beyond a critical value, $\tau_{c}$, where

$$
\tau_{c}>\frac{1}{2} \text { and }\left(2 \tau_{c}-1\right)^{2}=4 \mu D s .
$$

From (3.2) it can be seen that the critical wave number that goes unstable is

$$
k_{c}^{2}=\frac{2 \tau_{c}-1}{2 \mu D} .
$$

Note that $\operatorname{Im} \sigma\left(k_{c}^{2}\right) \neq 0$, therefore stability is lost through a Hopf bifurcation (see Fig. 1).

\section{Steady state analysis}

In Sect. 3 we showed that the non-trivial uniform steady state of the model system $(2.6 \mathrm{a}, \mathrm{b})$ lost stability through a Hopf bifurcation and therefore we would expect the system to exhibit spatio-temporal oscillations. However, if we now consider solving the system on the finite domain $[0,1]$ and impose the boundary conditions

$$
\frac{\partial n}{\partial x}=u=\frac{\partial^{2} u}{\partial x^{2}}=0
$$

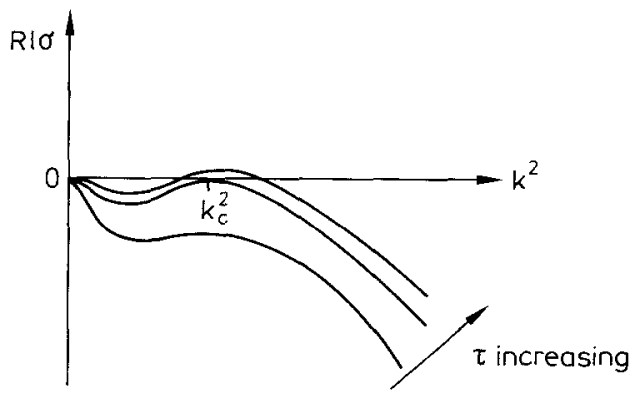

Fig. 1. Solution to (3.2) for $D>(D+\alpha) \tau$, $\tau>\frac{1}{2}$. As $\tau$ increases past a critical value $\tau_{c}$ (given by (3.3a)), the uniform steady state of $(2.6 a, b)$ loses stability through a Hopf bifurcation to disturbances of wave number $k_{c}$ (given by (3.3b)) 
at $x=0$ and $x=1$, then travelling wave type solutions are not permissible and the only types of heterogeneous solutions we would expect are purely spatial pattern or a temporally 'sloshing' pattern that satisfies (4.1) at the boundary but has temporal variation in the interior of the domain. In this section we restrict our attention to purely spatial pattern. Then, instability will arise only if a disturbance with wave number that satisfies the boundary conditions (4.1) has a temporal growth that is purely real and positive. The system may then evolve into a spatially heterogeneous steady state. Clearly, from the form of the dispersion relation (3.2) such a situation is possible. The steady state problem for $(2.6 \mathrm{a}, \mathrm{b})$ is

$$
\begin{gathered}
D \frac{d^{2} n}{d x^{2}}+\alpha \frac{d}{d x}\left(n \frac{d^{2} u}{d x^{2}}\right)=0 \\
\frac{d^{2} u}{d x^{2}}+\tau \frac{d}{d x}\left(n-n \frac{d u}{d x}\right)-s u\left(1-\frac{d u}{d x}\right)=0
\end{gathered}
$$

with boundary conditions (4.1) where, now, $n=n(x)$ and $u=u(x)$. We can integrate (4.2a) once and use (4.1) to get

$$
D \frac{d n}{d x}+\alpha n \frac{d^{2} u}{d x^{2}}=0
$$

which gives

$$
n=C \exp \left(-\frac{\alpha}{D} \frac{d u}{d x}\right)
$$

where $C$ is such that $\int_{0}^{1} n d x=n_{0}$, the total cell number. Substituting this expression for $n$ into (4.2b) we have

$$
\left[1-\tau C \exp \left(-\frac{\alpha}{D} \frac{d u}{d x}\right)\left\{1+\frac{\alpha}{D}-\frac{\alpha}{D} \frac{d u}{d x}\right\}\right] \frac{d^{2} u}{d x^{2}}+s u\left(\frac{d u}{d x}-1\right)=0 .
$$

Setting $p=d u / d x,(4.4)$ may be written as a pair of first order differential equations, namely,

$$
\begin{gathered}
\frac{d u}{d x}=p, \\
\frac{d p}{d x}=-\frac{s u(p-1)}{1-\tau C e^{-\alpha p / D}\left(1+\frac{\alpha}{D}-\frac{\alpha}{D} p\right)} .
\end{gathered}
$$

Figure 2 illustrates the phase plane for system (4.5). From this figure we can deduce the possible steady states. For example, the trajectory that starts at $A$, at $x=0$ and comes back to $A$ at $x=1$ automatically satisfies the boundary conditions because

$$
u=0, \quad \frac{d^{2} u}{d x^{2}}=\frac{d p}{d x}=0
$$

and

$$
\frac{d n}{d x}=-C \frac{\alpha}{D} \frac{d^{2} u}{d x^{2}} \exp \left(-\frac{\alpha}{D} \frac{d u}{d x}\right)=0
$$




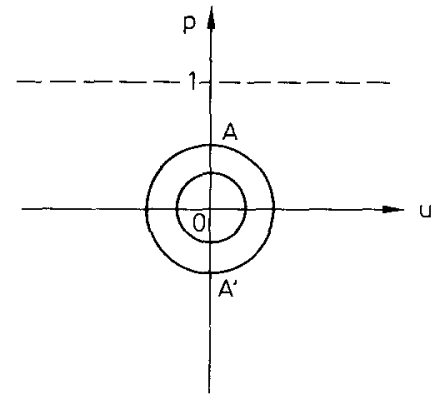

Fig. 2. Sketch of phase plane for (4.5). Note that the condition (2.6c) implies $p<1$

and is therefore a possible steady state. It is sketched in Fig. 3. Clearly, if the trajectory is traversed $m$ times we get $m$ humps for the cell density $n(x)$. These solutions are similar to those computed numerically in [8] for the time dependent problem for a more generalized system than the one we are studying here.

\section{Spatio-temporal oscillations}

If we do not impose boundary conditions (4.1) but instead impose periodic boundary conditions on $[0,1]$, then the linear analysis of Sect. 3 shows that the uniform steady state loses stability and may evolve to a travelling wave type solution. In this section we examine the time evolution of such a spatio-temporal oscillating instability using a multi-time scale perturbation procedure.

From Sect. 3, the uniform steady state loses linear stability as the cell traction parameter $\tau$ increases past a critical value $\tau_{c}$ given by (3.3a) to disturbances of wave number $k_{c}$ given by (3.3b). We shall assume that this disturbance satisfies the periodic boundary conditions. Clearly, we can choose $\mu$ and $D$ such that this is true. If we let

$$
\tau=\tau_{c}+\epsilon^{2} \delta, \quad 0<\epsilon \ll 1 \quad \text { and } \quad \delta= \pm 1 \text {, }
$$
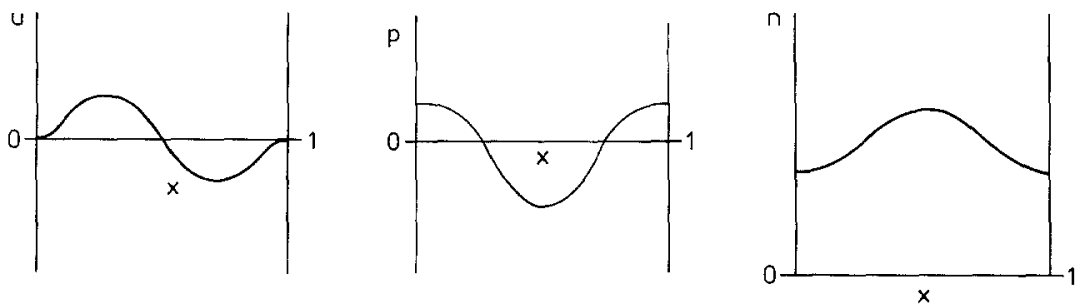

Fig. 3. Steady state corresponding to the phase trajectory from $A$ to $A$ in Fig. 2 . Note that if the trajectory went from $A$ to $A^{\prime}$ we would simply have a gradient solution for $n$, whereas if the trajectory $A$ to $A$ is traversed $m$ times we get a solution for $n(x)$ with $m$ humps. These solutions are similar to those computed in [8] for the time evolution problem for a more complicated version of the model 
then the expression $\exp \left\{\sigma t+i k_{c} x\right\}$ becomes

$$
\exp \left\{\epsilon^{2} t+i\left(\sigma_{I} t+k_{c} x\right)\right\} \quad \text { where } \sigma_{I}= \pm \sqrt{\frac{\left(D-(D+\alpha) \tau_{c}\right) k_{c}^{2}+s D}{\mu}} .
$$

We shall examine only the case where $\sigma_{I}$ is the positive solution in (5.2), that is, we look only for wave type solutions travelling in one direction. This suggests the change of coordinates

$$
T=\epsilon^{2} t, \quad z=x+\frac{\sigma_{l}}{k_{c}} t, \quad \partial_{t} \rightarrow \epsilon^{2} \partial_{T}+\frac{\sigma_{I}}{k_{c}} \partial_{z}, \quad \partial_{x} \rightarrow \partial_{z},
$$

where $T$ is a slow time scale and $z$ varies with $x$ and $t$. With this change of coordinates, system $(2.6 \mathrm{a}, \mathrm{b})$ becomes

$$
\begin{gathered}
\epsilon^{2} \frac{\partial n}{\partial T}+\frac{\sigma_{I}}{k_{c}} \frac{\partial n}{\partial z}+\frac{\partial}{\partial z}\left\{n\left(\epsilon^{2} \frac{\partial u}{\partial T}+\frac{\sigma_{I}}{k_{c}} \frac{\partial u}{\partial z}\right)\right\}=D \frac{\partial^{2} n}{\partial z^{2}}+\alpha \frac{\partial}{\partial z}\left(n \frac{\partial^{2} u}{\partial z^{2}}\right) \\
\mu\left(\epsilon^{2} \frac{\partial^{3} u}{\partial z^{2} \partial T}+\frac{\sigma_{I}}{k_{c}} \frac{\partial^{3} u}{\partial z^{3}}\right)+\frac{\partial^{2} u}{\partial z^{2}}+\left(\tau_{c}+\epsilon^{2} \delta\right)\left\{\frac{\partial n}{\partial z}-\frac{\partial}{\partial z}\left(n \frac{\partial u}{\partial z}\right)\right\} \\
=s u-s u \frac{\partial u}{\partial z}
\end{gathered}
$$

where, now, $n$ and $u$ are functions of $z, T$, and $\epsilon$. We expand $n$ and $u$ in the form

$$
\begin{gathered}
n(z, T, \epsilon)=1+\epsilon n_{1}(z, T)+\epsilon^{2} n_{2}(z, T)+\cdots, \\
u(z, T, \epsilon)=\epsilon u_{1}\left(z_{1}, T\right)+\epsilon^{2} u_{2}(z, T)+\cdots,
\end{gathered}
$$

and substitute into $(5.4 \mathrm{a}, \mathrm{b})$ and equate coefficients of $\epsilon$. At order $\epsilon$, we may write

$$
\begin{aligned}
& n_{1}(z, T)=a_{1}(T) \exp \left(i k_{c} z\right)+b_{1}(T) \exp \left(-i k_{c} z\right), \\
& u_{1}(z, T)=c_{1}(T) \exp \left(i k_{c} z\right)+d_{1}(T) \exp \left(-i k_{c} z\right),
\end{aligned}
$$

where $c_{1}(T)$ and $d_{1}(T)$ depend on $a_{1}(T)$ and $b_{1}(T)$. That is, we assume that the only mode that grows on the long time scale $T$ is that with wave number $k_{c}$ and that the solution is periodic in $z$, period $2 \pi / k_{c}$. Secular terms appear at order $\epsilon^{3}$. If we denote by $\mathscr{L}(n, u)$ the linearised operator from $(5.4 \mathrm{a}, \mathrm{b})$ and by $\underline{g}=\left(\begin{array}{l}g_{1} \\ g_{2}\end{array}\right)$ the secular terms that appear at $O\left(\epsilon^{3}\right)$, then a solution exists if and only if the Fredholm alternative (see [12], for example) is satisfied, that is

$$
\left\langle\underline{n}^{*}, g\right\rangle=\int_{0}^{2 \pi / k_{c}}\left(n^{*} \bar{g}_{1}+u^{*} \bar{g}_{2}\right) d z=0
$$

where the bar denotes the complex conjugate and $\underline{n}^{*}=\left(\begin{array}{l}n^{*} \\ u^{*}\end{array}\right)$ is the solution of $\mathscr{L}^{*}\left(n^{*}, u^{*}\right)=0$ where $\mathscr{L}^{*}$ is the adjoint operator to $\mathscr{L}(n, u)$. Solving (5.7) gives, 
after much tedious algebra, the evolution of $a_{1}$ and $b_{1}$ on the slow time scale as

$$
\begin{aligned}
& \frac{d a_{1}}{d T}=\delta P a_{1}+Q\left|a_{1}\right|^{2} \bar{a}_{1}, \\
& \frac{d b_{1}}{d T}=\delta R b_{1}+S\left|b_{1}\right|^{2} \bar{b}_{1},
\end{aligned}
$$

where $P, Q, R$, and $S$ are algebraic expressions involving the parameters $\alpha, D, \tau_{c}$, $\mu$ and $s$.

A realistic biological solution must have $n$, the cell density, real; therefore from (5.6a), we must have $b_{1}(T)=\bar{a}_{1}(T)$. This also makes $d_{1}(T)=\bar{c}_{1}(T)$ ensuring that $u$ is real, too. With this substitution, $R=\bar{P}$ and $S=\bar{Q}$ in (5.8). If we multiply ( $5.8 \mathrm{a}$ ) by $\bar{a}_{1}$ and ( $5.8 \mathrm{~b}$ ) by $a_{1}$ and add the resulting equations we find the evolution equation for $\left|a_{1}\right|^{2}$ :

$$
\frac{d}{d T}\left|a_{1}\right|^{2}=\delta(2 R \ell P)\left|a_{1}\right|^{2}+2 R \ell Q\left|a_{1}\right|^{4}
$$

where $P$ and $Q$ are given in Appendix A.2.

We summarize the behaviour of (5.9) in Table 1. Thus in the appropriate parameter space, we have the small amplitude solution to order $\epsilon$,

$$
\begin{gathered}
n(x, t, T)=1+\epsilon 2 R l a_{1}(T) \cos \left(k_{c} x+\sigma_{I} t\right), \\
u(x, t, T)=\epsilon 2 R l c_{1}(T) \cos \left(k_{c} x+\sigma_{I} t\right),
\end{gathered}
$$

where

$$
c_{1}(T)=\left\{\frac{D k_{c}^{2}+i \sigma_{I}}{\sigma_{I} k_{c}^{2}-i \alpha k_{c}^{3}}\right\} a_{1}(T) .
$$

A typical solution is sketched in Fig. 4. From Table 1 we see the possibility of a subcritical bifurcation. If $\delta<0$ and $R l Q>0$, then small disturbances die out but larger ones grow. This temporal growth is unbounded and our power series expansion (5.5) becomes invalid.

Table 1. Summary of the solution to (5.9). Note that, for example, if $\delta>0$, $R l Q>0$, then $a_{1}(T)$ is unbounded. Thus, in this parameter regime our power series expansion (5.5) is invalid

\begin{tabular}{lll}
\hline$R l Q>0$ & $\frac{\delta<0}{}$ & \\
& $\left|a_{1}\right|^{2} \rightarrow \infty$ & if $\left|a_{1}(0)\right|^{2}<\sqrt{\frac{R l P}{R l Q},} \quad\left|a_{1}\right|^{2} \rightarrow 0$ \\
$R l Q<0$ & if $\left|a_{1}(0)\right|^{2}>\sqrt{\frac{R l P}{R l Q}}, \quad\left|a_{1}\right|^{2} \rightarrow \infty$ \\
& $\left|a_{1}\right|^{2} \rightarrow \sqrt{\frac{R l P}{|R l Q|}}$ & $\left|a_{1}\right|^{2} \rightarrow 0$
\end{tabular}




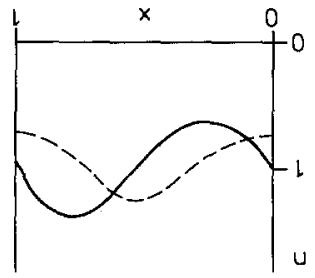

Fig. 4. Sketch of the first harmonic of the spatio-temporally oscillating solution (5.10) for cell density $n$ where $k_{c}=2 \pi$. This is similar to the solutions computed numerically in [8] for a more complex version of the model

In the expansion (5.5), the terms $n_{2}(z, T)$ and $u_{2}(z, T)$ contain second harmonics, that is, terms of the form $\exp \left(2 i k_{c} z\right)$ and $\exp \left(-2 i k_{c} z\right)$. These may be easily calculated from (5.10). (See Appendix A.3.)

\section{Singular dispersion relation}

In [7] it was shown that in various parameter spaces the general model of Oster et al. exhibited a singular dispersion relation when linearised about the uniform non-zero steady state. That is, the initial growth rate of a disturbance of wave number $k$ tended to positive or negative infinity as $k$ tended to some critical wave number. Britton [13] analysed a singular dispersion relation arising in a one equation caricature of the original model. In this section we analyse one of the actual models discussed in [5] that has a singular dispersion relation. If we set $\mu=0$ in (2.6b), that is, if we assume that the ECM behaves as a purely elastic medium and that the elastic restoring forces generated by the ECM are in mechanical balance with cell traction, then the model system reduces to

$$
\begin{gathered}
\frac{\partial n}{\partial t}+\frac{\partial}{\partial x}\left(n \frac{\partial u}{\partial t}\right)=D \frac{\partial^{2} n}{\partial x^{2}}+\alpha \frac{\partial}{\partial x}\left(n \frac{\partial^{2} u}{\partial x^{2}}\right) \\
\frac{\partial^{2} u}{\partial x^{2}}+\tau \frac{\partial}{\partial x}\left(n-n \frac{\partial u}{\partial x}\right)=s u\left(1-\frac{\partial u}{\partial x}\right)
\end{gathered}
$$

with boundary conditions

$$
\frac{\partial n}{\partial x}=u=\frac{\partial^{2} u}{\partial x^{2}}=0 \quad \text { at } x=0,1
$$

From (3.2) with $\mu=0$, the dispersion relation is

$$
\sigma\left(k^{2}\right)=-\frac{\{D-(D+\alpha) \tau\} k^{4}+s D k^{2}}{(1-2 \tau) k^{2}+s} .
$$

If $\tau$ satisfies the condition

$$
\tau>\max \left(\frac{1}{2}, \frac{D}{D+\alpha}\right),
$$

then

$$
\sigma=0 \quad \text { at } k_{0}^{2}=\frac{s D}{(D+\alpha) \tau-D}, \quad \sigma \rightarrow \infty \quad \text { at } k_{c}^{2}=\frac{s}{2 \tau-1} \quad(\text { if } D \neq \alpha) .
$$


If $D=\alpha$, then $\sigma\left(k^{2}\right)=-D k^{2}$ and the uniform steady state is linearly stable. The behaviour of $\sigma\left(k^{2}\right)$ as $\alpha$ varies is sketched in Fig. 5 .

We shall assume that there is one and only one wave number $k$ that satisfies (6.1c) and has positive temporal growth rate. Furthermore, we assume that $k=k_{c}$.

In Sect. 5, the linear growth rate was small, so we looked for solutions that evolved on the slow time scale $\epsilon^{2} t$. In this case, the linear growth rate is very large and suggests a fast-focussing effect. Thus we should look for solutions that evolve on the fast time scale $t / \epsilon$. Therefore we set

$$
\alpha_{c}=D, \quad \alpha=\alpha_{c}-\delta \epsilon \quad \text { where } 0<\epsilon \ll 1, \quad \delta= \pm 1, \quad \text { and } T=t / \epsilon .
$$

We ignore variation on the normal time scale $t$, therefore $n, u$ are functions of $x, T, \epsilon$ and on substituting (6.4) into (6.1a, b) we obtain

$$
\begin{gathered}
\frac{\partial n}{\partial T}+\frac{\partial}{\partial x}\left(n \frac{\partial u}{\partial T}\right)=\epsilon\left[D \frac{\partial^{2} u}{\partial x^{2}}+\alpha_{c}\left(n \frac{\partial^{2} u}{\partial x^{2}}\right)\right]-\epsilon^{2} \delta \frac{\partial}{\partial x}\left(n \frac{\partial^{2} u}{\partial x^{2}}\right), \\
\frac{\partial^{2} u}{\partial x^{2}}+\tau \frac{\partial}{\partial x}\left(n-n \frac{\partial u}{\partial x}\right)=s u\left(1-\frac{\partial u}{\partial x}\right) .
\end{gathered}
$$

We expand $n$ and $u$ in the form

$$
\begin{gathered}
n(x, T, \epsilon)=1+\epsilon n_{1}(x, T)+\epsilon^{2} n_{2}(x, T)+\cdots, \\
u(x, T, \epsilon)=\epsilon u_{1}(x, T)+\epsilon^{2} u_{2}(x, T)+\cdots,
\end{gathered}
$$

and substitute into $(6.5 \mathrm{a}, \mathrm{b})$ and equate coefficients of $\epsilon$.

The order $\epsilon$ terms give

$$
\begin{gathered}
\frac{\partial n_{1}}{\partial T}+\frac{\partial}{\partial T}\left(\frac{\partial u_{1}}{\partial x}\right)=0, \\
(1-\tau) \frac{\partial^{2} u_{1}}{\partial x^{2}}-s u_{1}+\tau \frac{\partial n_{1}}{\partial x}=0 .
\end{gathered}
$$

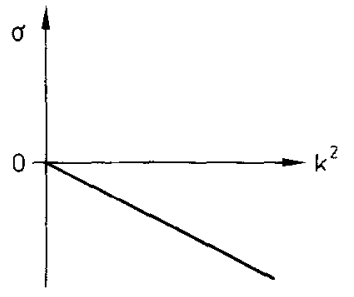

a

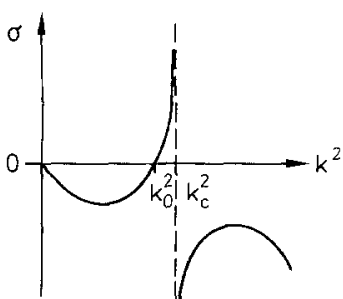

b

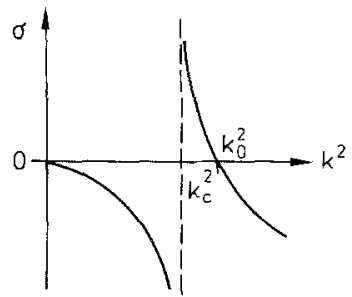

C

Fig. 5a-c. The behaviour of $\sigma\left(k^{2}\right)$ (Eq. (6.2)) as $\alpha$ varies, with $\tau$ satisfying the conditions (6.3). Note that if $\alpha \neq D$ the system (6.1) loses linear stability and exhibits singular behaviour. ( $k_{c}^{2}$ and $k_{0}^{2}$ are given in the text). a $\alpha=D ; \mathbf{b} \alpha=D+\epsilon ; \mathbf{c} \alpha=D-\epsilon$ 
We can integrate $(6.7 \mathrm{a})$ with respect to $T$ to get

$$
n_{1}+\frac{\partial u_{1}}{\partial x}=F(x)
$$

where $F(x)$ is an arbitrary function of $x$. The solution to $(6.7 \mathrm{~b}, \mathrm{c})$ with boundary conditions (6.1c) is

$$
\begin{gathered}
n_{1}=a_{1}(T) \cos k_{c} x+n_{\rho}(x), \\
u_{1}=b_{1}(T) \sin k_{c} x+u_{\rho}(x), \text { where } a_{1}=-k_{c} b_{1},
\end{gathered}
$$

and $\left(n_{1}, u_{1}\right)=\left(n_{\rho}, u_{\rho}\right)$ is a particular solution to $(6.7 \mathrm{c})$ satisfying $(6.1 \mathrm{c})$. Our asymptotic procedure assumes that on the $T$ time scale the system has isolated the spatial disturbance of wave number $k_{c}$ and that this is the only spatial dependence of the solution (see, for example [14]). Thus, in order to be consistent, we must take $n_{\rho}(x) \equiv u_{\rho}(x) \equiv 0$. This forces $F(x) \equiv 0$.

At $O\left(\epsilon^{2}\right)$ a secular term appears in the first equation of the form

$$
D \frac{\partial^{2} n_{1}}{\partial x^{2}}+\alpha_{c} \frac{\partial^{3} u_{1}}{\partial x^{3}}
$$

However, because of the special choice of $k_{c}$ this term is identically zero. Secular terms appear at $O\left(\epsilon^{3}\right)$ and may be suppressed as described in Sect. 4. This gives rise to the amplitude equation

$$
\frac{d a_{1}}{d T}=\frac{1}{a_{1}}\left[-\delta X+Y a_{1}^{2}\right],
$$

where

$$
X=4 k_{c}^{2}, \quad Y=\frac{1}{\tau(2 \tau+1)}\left[17 \tau-3-2 \tau^{2}\right]
$$

\begin{tabular}{|c|c|c|}
\hline & $\delta>0$ & $\delta<0$ \\
\hline$Y>0$ & if $a_{1}(0)<\sqrt{\frac{X}{Y}}, \quad a_{1} \rightarrow 0$ & $a_{1} \rightarrow \infty$ \\
\hline & if $a_{1}(0)>\sqrt{\frac{X}{Y}}, \quad a_{1} \rightarrow \infty$ & \\
\hline$Y<0$ & $a_{1} \rightarrow 0$ & $a_{1} \rightarrow \sqrt{\frac{X}{|Y|}}$ \\
\hline
\end{tabular}

The solution to (6.9) is summarized in Table 2. Clearly, in the appropriate parameter space, $n$ and $u$ evolve to the small amplitude solution to first

Table 2. Summary of the solution to (6.9) 
order in $\epsilon$,

$$
\begin{aligned}
& n=1+\epsilon \sqrt{\frac{X}{|Y|}} \cos k_{c} x, \\
& u=-\epsilon \frac{1}{k_{c}} \sqrt{\frac{X}{|Y|}} \sin k_{c} x,
\end{aligned}
$$

if $\delta<0, Y<0$. The condition $Y<0$ implies $2 \tau^{2}-17 \tau+3>0$. That is,

$$
\tau>\frac{17+\sqrt{265}}{4} \text { or } \tau<\frac{17-\sqrt{265}}{4} .
$$

The second inequality contradicts condition (6.3); therefore we have the conditions

$$
\tau>\frac{17+\sqrt{265}}{4} \text { and } \alpha=D+\epsilon
$$

where the second condition comes from substituting $\delta=-1$ into (6.4).

\section{Discussion}

We have analysed a cell-haptotaxis model and have shown that it may exhibit spatial and spatio-temporal patterns. The solutions obtained from the nonlinear analyses are similar to those computed numerically in [8]. The spatial patterns are similar to those obtained by Grindrod et al. [15], for a cell-chemotaxis model. However, the model analysed here can give rise to spatio-temporal oscillations, too. This type of behaviour has been exploited by Goldwasser et al. [16], to show how temporal oscillations may account for certain cell splitting and branching phenomena. The full analysis of the spatio-temporal case is a formidable task and we have made several simplifying assumptions in Sect. 5. We have assumed that the wave moves only in one direction, that is, we have ignored the negative solution for $\sigma_{I}$ in (5.2). Including this root would lead to waves travelling in opposite directions and thus there would be two pairs of amplitude equations and the possibility of standing waves. Thus the validity of our analysis is restricted to situations in which a direction is specified. Biologically, this may correspond to a case where cells are introduced at one end of a domain and propagate through it.

The second major assumption is that we look at monochromatic waves, that is, waves of one wave length in space. More generally, this type of propagating wave problem should consider the motion of a wave packet. The interaction of several spatially varying perturbations suggests a slowly varying spatial coordinate (see, for example, [17]) and calculation of a group velocity. However, the complex form of the equations and the dispersion relation in this problem makes such an analysis formidable algebraically and here we have examined the time evolution of a particular periodic pattern. We intend to analyse a simpler version of the general model [5] to extend the analysis presented in Sect. 5 and to address the above points more fully. 
We also presented a formal analysis for the case of a singular dispersion relation and showed that, in the appropriate parameter space, the system could evolve to a small amplitude spatially heterogeneous steady state.

In all the above analyses, we delimited parameter regimes wherein certain phenomena will occur. Although all the parameters are in principle measureable experimentally, it is difficult to obtain accurate measurements. An advantage of non-dimensionalising the system is that we deal with parameter groupings and it is easier to compare, quantitatively, relative sizes of parameters. For example the analysis in Sect. 3 shows that pattern formation occurs if the non-dimensional cell traction parameter increases beyond a critical value, and (6.11) shows that fast-focussing is possible for very large cell traction. Cell traction has been measured for mesenchymal cells in vitro [18] and such cells can exert very large traction [19]. It is also known that cell traction in vitro increases abruptly over the first few days in culture (Harris, personal communication). Such behaviour reinforces the possibility of cell traction playing the role of the bifurcation parameter. Of course, this can be said only of the in vitro case and more experimental work is necessary before one can extend this to the in vivo situation.

\section{Appendix}

A.1. Equations (2.1), (2.2) and (2.3) may be non-dimensionalised as follows: let $L$ and $T_{0}$ be typical length and time scales respectively, $N$ be average cell density and $\rho_{0}$ be average extracellular matrix density.

We define non-dimensional (tilde) variables as

$$
\begin{gathered}
\bar{t}=\frac{t}{T_{0}}, \quad \underline{\tilde{x}}=\frac{\underline{x}}{L}, \quad \tilde{n}=\frac{n}{N}, \quad \tilde{\rho}=\frac{\rho}{\rho_{0}}, \quad \underline{\tilde{u}}=\frac{\underline{u}}{L}, \\
\tilde{D}=\frac{D T_{0}}{L^{2}}, \quad \tilde{s}=\frac{s \rho_{0}(1+v) L^{2}}{E}, \quad \tilde{\alpha}=\frac{\alpha \rho_{0} T_{0}}{L^{2}}, \quad \tilde{\mu}_{1}=\frac{(1+v) \mu_{1}}{E T_{0}}, \\
\tilde{\mu}_{2}=\frac{(1+v) \mu_{2}}{E T_{0}}, \quad \tilde{\tau}=\frac{\tau N \rho_{0}(1+v)}{E} .
\end{gathered}
$$

This non-dimensionalisation transforms the one-dimensional version of (2.1), (2.2) and (2.3) into equations (2.4), where we have dropped the tildes for notational convenience.

A.2. In Eq. (5.9), $P$ and $Q$ are given by the following expressions:

$$
P=\frac{P_{1}}{P_{2}}, \quad Q=\frac{Q_{1}}{Q_{2}},
$$

where

$$
\begin{gathered}
P_{1}=k_{c}\left\{(3 D+\alpha) k_{c} V+i\left(2 V^{2}-D k_{c}^{2}(D+\alpha)\right)\right\} \\
P_{2}=-2 \mu D k_{c}^{2} V+i k_{c}\left(\mu D^{2} k_{c}^{2}+(\alpha-D) \tau_{c}-\mu V^{2}\right), \\
Q_{1}=Q_{11} \overline{Q_{13}}\left\{-2 V \tau_{c} k_{c}^{2}+i(D+\alpha) k_{c}^{3}\right\}+2 k_{c}^{2} \tau Q_{12}\left\{2 V-i(2 \alpha+D) k_{c}\right\} \\
+i k_{c} s \overline{Q_{13}}\left(V-i D k_{c}\right), \\
Q_{2}=\frac{P_{1}}{V-i \alpha k_{c}},
\end{gathered}
$$




$$
\begin{gathered}
V=\frac{\sigma_{I}}{k_{c}}, \\
Q_{11}=\frac{Q_{111} Q_{112}-Q_{113} Q_{114}}{Q_{115} Q_{111}-Q_{113} Q_{116}}, \\
Q_{12}=\frac{Q_{114} Q_{115}-Q_{116} Q_{112}}{Q_{115} Q_{111}-Q_{113} Q_{116}}, \\
Q_{13}=\frac{D k_{c}+i V}{V k_{c}-i \alpha k_{c}^{2}}, \\
Q_{111}=4 k_{c}^{2}\left(\tau_{c}-1\right)-s-i 8 \mu V k_{c}^{3}, \\
Q_{112}=2 k_{c}\left(D k_{c}+i V\right) \\
Q_{113}=4 k_{c}^{2}\left(-V+i 2 k_{c} \alpha\right), \\
Q_{114}=-k_{c} Q_{13}\left(2 k_{c} \tau_{c}+i s Q_{13}\right), \\
Q_{115}=2 k_{c}\left(2 k_{c} D+i V\right), \\
Q_{116}=i k_{c} \tau_{c}, \\
k_{c}^{2}=\frac{2 \tau_{c}-1}{2 \mu D} .
\end{gathered}
$$

A.3. In (5.5) $n_{2}(z, T)$ and $u_{2}(z, T)$ have the form

$$
\begin{aligned}
n_{2}(z, T)= & a_{2}(T) \exp \left(i k_{c} z\right)+b_{2}(T) \exp \left(-i k_{c} z\right)+a_{21}(T) \exp \left(2 i k_{c} z\right) \\
& +b_{21}(T) \exp \left(-2 i k_{c} z\right) \\
u_{2}(z, T)= & c_{2}(T) \exp \left(i k_{c} z\right)+d_{2}(T) \exp \left(-i k_{c} z\right)+c_{21}(T) \exp \left(2 i k_{c} z\right) \\
& +d_{21}(T) \exp \left(-2 i k_{c} z\right)
\end{aligned}
$$

Making the assumption that $b_{1}(T)=\bar{a}_{1}(T)$ automatically ensures that the coefficients of the second harmonics are also complex conjugate pairs, that is, $b_{21}=\bar{a}_{21}$ and $d_{21}=\bar{c}_{21}$, where $a_{21}=Q_{11} a_{1}^{2}, c_{21}=Q_{12} a_{1}^{2}$ and the second harmonic takes the form (at $\left.O\left(\epsilon^{2}\right)\right) \quad 2 R l\left(Q_{11} a_{1}^{2}(T)\right) \cos \left(2\left(k_{c} x+\sigma_{I} t\right)\right)$ and $2 R l\left(Q_{12} a_{1}^{2}(T)\right) \cos \left(2\left(k_{c} x+\sigma_{I} t\right)\right)$ for $n$ and $u$, respectively.

Acknowledgements. I would like to thank Professor J. D. Murray, Centre for Mathematical Biology, University of Oxford, for helpful discussions. I also thank Annetta Cochran for typing the manuscript.

\section{References}

1. Turing, M. A.: The chemical basis of morphogenesis. Philos. Trans. R. Soc. Lond., B 237, 37-73 (1952)

2. Murray, J. D.: A pre-pattern formation mechanism for animal coat markings, J. Theor. Biol. 88, $161-199$ (1981)

3. Meinhardt, H.: Models of biological pattern formation. London: Academic Press 1982

4. Wolpert, L.: Positional information and the spatial pattern of cellular differentiation, J. Theor. Biol. 25, 1-47 (1969)

5. Oster, G. F., Murray, J. D., Harris, A. K.: Mechanical aspects of mesenchymal morphogenesis. J. Embryol. Exp. Morph. 78, 83-125 (1983) 
6. Oster, G. F., Murray, J. D., Maini, P. K.: A model for chondrogenic condensations in the developing limb: the role of extracellular matrix and cell tractions. J. Embryol. Exp. Morph. 89, 93-112 (1985)

7. Murray, J. D., Oster, G. F.: Cell traction models for generating pattern and form in morphogenesis. J. Math. Biol. 19, 265-279 (1984)

8. Perelson, A. S., Maini, P. K., Murray, J. D., Hyman, J. M., Oster, G. F.: Nonlinear pattern selection in a mechanical model for morphogenesis. J. Math. Biol. 24, 525-541 (1986)

9. Maini, P. K., Murray, J. D.: A nonlinear analysis of a mechanical model for biological pattern formation. SIAM J. Appl. Math. 48, 1064-1072 (1988)

10. Haken, H.: Synergetics. An introduction. Nonequilibrium phase transitions and self-organisation in physics, chemistry and biology. Berlin Heidelberg New York: Springer 1977

11. Fife, P. C.: Mathematical aspects of reacting and diffusing systems. Berlin Heidelberg New York: Springer 1979

12. Keener, J. P.: Principles of applied mathematics, transformation and approximation. Reading, Mass.: Addison-Wesley 1988

13. Britton, N. F.: A singular dispersion relation arising in a caricature of a model for morphogensis. J. Math. Biol. 26, 387-403 (1988)

14. Matkowsky, B. J.: A simple non-linear dynamic stability problem. Bull. Am. Math. Soc. 76, 620-625 (1970)

15. Grindrod, P., Sinha, S., Murray, J. D.: Steady state spatial patterns in a cell-chemotaxis model. I.M.A. J. Math. Appl. Med. Biol. (in press)

16. Goldwasser, L., Maini, P. K., Murray, J. D.: Splitting of cell clusters and bifurcation of bryozoan branches. J. Theor. Biol. 137, 271-279 (1989)

17. Stewartson, K., Stuart, J. T.: A non-linear instability theory for a wave system in plane Poiseuille flow. J. Fluid Mech. 48, 529-545 (1971)

18. Harris, A., Stopak, D., Wild, P.: Fibroblast traction as a mechanism for collagen morphogenesis. Nature 290, 249-251 (1981)

19. Harris, A., Wild, P., Stopak, D., Silicone rubber substrata: a new wrinkle in the study of cell locomotion. Science 208, 177--179 (1980)

Received January 16, 1989/Revised March 27, 1989 